\title{
Approche techno-typologique des peignes à tatouer en nacre polynésiens. Un moyen d'appréhender la pensée technique
}

\section{Guillaume Molle et Éric Conte}

\author{
(2) OpenEdition \\ Journals \\ Édition électronique \\ URL : http://journals.openedition.org/jso/7019 \\ DOI : $10.4000 /$ jso.7019 \\ ISSN : $1760-7256$ \\ Éditeur \\ Société des océanistes \\ Édition imprimée \\ Date de publication : 15 octobre 2013 \\ Pagination : 209-225 \\ ISBN : 978-2-85430-035-2 \\ ISSN : 0300-953x
}

Référence électronique

Guillaume Molle et Éric Conte, « Approche techno-typologique des peignes à tatouer en nacre polynésiens. Un moyen d'appréhender la pensée technique », Journal de la Société des Océanistes [En ligne], 136-137 | 2013, mis en ligne le 14 novembre 2013, consulté le 16 avril 2020. URL : http:// journals.openedition.org/jso/7019; DOI : https://doi.org/10.4000/jso.7019 


\title{
Les peignes à tatouer en nacre polynésiens. Un moyen d'appréhender la pensée technique
}

par

\author{
Guillaume MOLLE* et Éric CONTE*
}

\section{RÉSUMÉ}

L'étude technologique d'une collection de 17 peignes à tatouer en nacre, découverts en contexte archéologique dans trois archipels de Polynésie française, offre une reconstitution des processus de fabrication intervenant dans la chaîne opératoire et permet aussi d'intégrer ces objets originaux aux typologies existantes. Les peignes en nacre sont également replacés pour la première fois dans une perspective diachronique qui ouvre de nouvelles voies de réflexion quant au modèle de développement régional de ces artefacts.

MoTs-CLÉs : tatouage, peignes, nacre, technologie, Polynésie

La découverte de trois peignes à tatouer fabriqués en nacre lors de la fouille du site de Hane en 2009 (Molle, 2011 : 114) nous a amenés à nous interroger sur la nature technique de ces objets relativement rares en contexte archéologique et absents des descriptions ethnohistoriques. Une recherche approfondie nous a conduits à établir un corpus quasi-exhaustif des objets de ce type connus, soit 17 pièces provenant de différents sites fouillés dans trois archipels de Polynésie française (Marquises, Société, Australes). En fonction de leur lieu de conservation, certaines pièces ont pu être étudiées plus précisément, d'autres n'ont été observées que grâce à des photographies.

Cet article a pour but d'éclairer la place de ces artefacts originaux dans la panoplie matérielle

\begin{abstract}
We present here a technological study of a collection of 17 archaeological pearl-shell tattooing combs. They have been discovered in three archipelagoes of French Polynesia. The study offers a reconstitution of the manufacturing processes involved in the "chaîne operatoire". Then, the combs are integrated to the existing typologies. For the first time, we also put them back in a diachronic perspective that allows new reflections about a model of regional development of these artifacts.
\end{abstract}

KeYwords: tattooing, combs, pearl-shell, technology, Polynesia

des anciens Polynésiens. Nous nous intéresserons plus particulièrement à l'analyse technologique des pièces permettant de reconstituer la chaîne opératoire de fabrication ainsi qu'à leurs formes, offrant ainsi une première perspective typologique. Un essai de synthèse quant au développement des formes en Polynésie orientale clôturera notre exposé.

\section{Présentation du corpus}

Dix-sept peignes à tatouer en nacre, dont 14 recueillis en contextes archéologiques dans trois archipels de Polynésie française, composent notre corpus d'étude.

\footnotetext{
* Centre international de recherche archéologique sur la Polynésie (CIRAP - UPF), UMR 7041 ArScAn (Équipe Ethnologie préhistorique), LabEx CORAIL, guillaume.molle@upf.pf, conte@upf.pf
} 


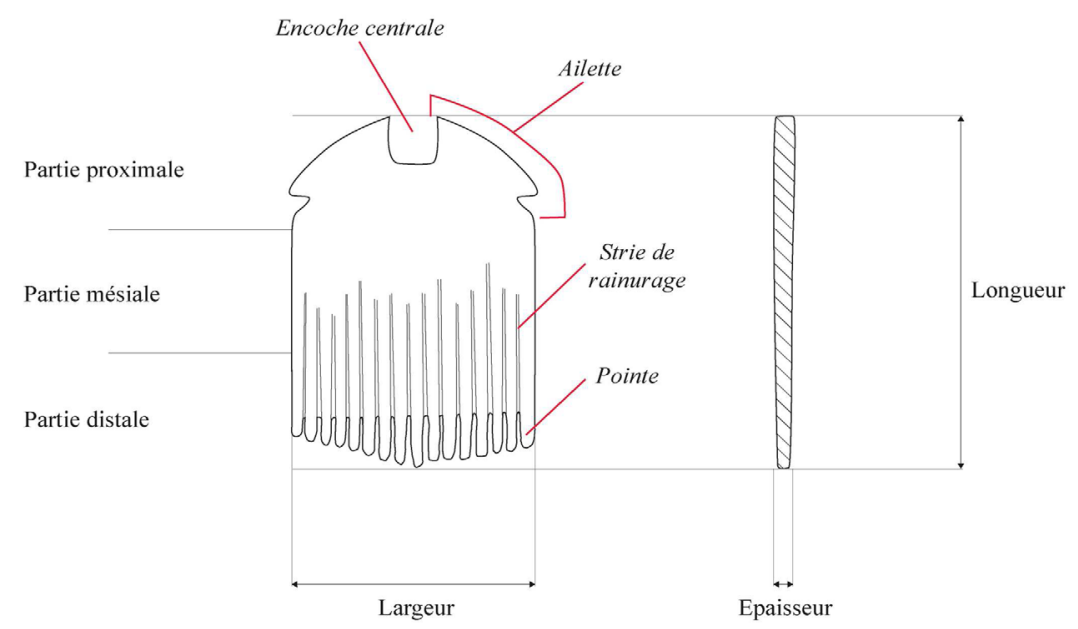

Figure 1. - Schéma descriptif d'un peigne à tatouer

Dix peignes proviennent du groupe Nord des Marquises et plus particulièrement de l'île de Ua Huka. Six d'entre eux ont été découverts lors de la fouille du site dunaire de Hane en 196465 dirigée par Y. Sinoto (Sinoto et Kellum, 1965) et en partie présentés dans un article de synthèse (Sinoto, 1966 : 298, fig.4b). Notons que sur les planches publiées apparaissent des peignes à tatouer en os, ici hors de notre propos. De plus, Sinoto interprétait l'une des pièces (H86-4) comme un possible instrument de tatouage à bord coupant ayant pu servir à entailler la peau, tels les peignes maori nommés uhi tapabi en Nouvelle-Zélande (Best, 1904 : 167 ; Sinoto, com. pers., déc. 2010). Toutefois, nous n'accordons que peu de crédit à cette hypothèse et il nous semble plus juste de considérer cet objet comme l'ébauche d'un pendentif en nacre dont d'ailleurs d'autres exemplaires sont connus, tant à Hane que sur le site de Vaito'otia à Huahine (Sinoto, 1983). Cette conclusion fut aussi récemment proposée par Ambrose (2012 : 5). En 2009, le site de Hane fit l'objet d'une nouvelle campagne de fouille sous la direction de É. Conte et P. Murail (cf. Molle, 2011). À cette occasion ont été découverts trois peignes en nacre qui s'ajoutent donc aux précédents. Un autre exemplaire fut recueilli dans les années 1990 par Marie-Josée Vaatete lors de la construction d'une maison dans la partie sommitale de la dune de Hane, à l'entrée de l'actuel village. Enfin, un peigne fut découvert sur le site dunaire de Ha'atuatua à Nuku Hiva par B. Rolett et É. Conte lors de la reprise des fouilles de Suggs (Rolett et Conte, 1995 : 222, fig. 15). Le corpus marquisien s'élève ainsi à 11 pièces.

Aux îles de la Société, on compte deux peignes en nacre provenant du site de Vaito'otia à Huahine fouillé par Sinoto (1983 : 588, fig.4). Un troisième fut découvert lors des fouilles de sauvetage organisées sur le site de Rivnac à Punaauia, Tahiti (Eddowes et Dennison, 1996 : 6, fig.4b).

Enfin, nous connaissons trois peignes en nacre dans l'archipel des Australes. Tous proviennent de la côte Nord de l'île de Tubuai. L'un fut recueilli par R. Bollt en 2007 sur le site de Atiahara sur lequel aucune synthèse n'a été publiée à ce jour. Deux autres exemplaires ont été collectés en surface sur des parcelles voisines de Atiahara par Larry Miller, un habitant de l'île qui a participé à la campagne de 2007, et sont depuis conservés chez lui.

Les objets sont aujourd'hui dispersés dans plusieurs collections dont celles du Musée de Tahiti et des Îles, du Service de la Culture et du Patrimoine de Tahiti et du laboratoire du CIRAP à l'Université de la Polynésie française. Nous avons été en mesure d'étudier ces pièces en détail. Cinq peignes de Hane et de Vaito'otia sont conservés au Bishop Museum de Hawaii. Le Dr. Sinoto a très aimablement accepté de nous transmettre ses clichés ainsi que quelques informations complémentaires sur leurs dimensions. Le peigne recueilli en surface à Hane est conservé au musée communal de Vaipaee, à Ua Huka. Celui découvert à Ha'atuatua est présenté dans la salle patrimoniale de Hatiheu à Nuku Hiva. Pour celui-ci ainsi que pour les exemplaires de Atiahara toujours conservés à Tubuai, nous disposions des photographies comme documents de travail, à partir desquelles des dessins et des observations ont été réalisés.

\section{Terminologie descriptive}

Tous les peignes recueillis sont taillés dans des valves de l'huître perlière Pinctada margaritifera. Les objets se présentent à divers stades de fabri- 
cation, allant d'une plaquette de nacre peu travaillée à la pièce achevée, voire même cassée suite à son utilisation. Afin de faciliter leur description, il convient d'employer une terminologie normalisée indiquée sur la figure 1.

Par convention, nous considérerons la partie proximale de la pièce comme celle la plus proche du praticien lors de l'opération de tatouage. Il s'agira donc du sommet du peigne recevant divers aménagements pour la fixation au manche de bois. Sur le schéma, nous indiquons l'encoche centrale et les ailettes latérales qui constituent le système le plus commun dans notre corpus, le second mode reconnu étant celui de la simple perforation centrale. La partie distale opposée correspond à l'extrémité du peigne dans laquelle sont façonnées les pointes (parfois appelées " dents " dans la littérature) qui pénétreront directement l'épiderme du patient au cours de l'opération. Chaque peigne est caractérisé par sa longueur, sa largeur et son épaisseur, ces dernières mesures pouvant varier entre les parties proximales et distales.

L'analyse des traces de fabrication repose sur l'emploi des termes définis pour les hameçons polynésiens (Carlier, 2002 ; Conte et Carlier, 2010) et dont les techniques de façonnage sont très semblables de par l'usage du même matériau. De plus, nous adoptons la même distinction entre les deux faces. La face interne désigne la partie interne de la valve huîtrière qui est aussi la plus nacrée, tandis que la face externe correspond à l'enveloppe extérieure de la valve aussi appelée periostracum. Bien que les procédés d'abrasion décrits plus loin aient en partie fait disparaître cette enveloppe, il est possible de distinguer les deux faces par la couleur et le brillant de la nacre. Si tel n'est pas le cas, les deux faces sont désignées de manière arbitraire.

Les descriptions des 16 peignes de notre corpus sont proposées en annexe de cette étude.

\section{Reconstitution du processus de fabrication}

L'analyse technologique des objets découverts à différents stades de fabrication nous offre pour la première fois l'opportunité de définir les étapes de la chaîne opératoire suivie pour le façonnage de ces pièces, représentées schématiquement sur la figure 2. Des éléments de comparaison et de classification enrichiront nos propos.

\section{Préparation du support de nacre et mise en forme générale}

La première étape consiste à découper dans la valve d'une huître perlière une plaquette de nacre qui servira à la confection du peigne. On suppose que la plupart des supports ont été découpés dans la partie centrale de la valve, à l'instar du peigne $\mathrm{n}^{\circ} \mathrm{TP} 12-4$ de Ha'atuatua qui présente sur sa face interne la zone d'attache du muscle adducteur. Seul l'exemplaire n ${ }^{\circ} \mathrm{O} 110-32$ de Hane, qui d'ailleurs constitue un cas à part dans notre corpus, est issu de la zone plus épaisse de la valve à proximité de la charnière.

La plaquette est probablement découpée dans un premier temps par rainurage bifacial dont on retrouve les traces sur plusieurs pièces. Néanmoins, il n'est pas exclu que les rainures observables témoignent d'une opération de rainurage secondaire destinée à préciser la forme du peigne. Si le recours au rainurage ne fait aucun doute, on peut également supposer que la percussion était utilisée de la même manière que pour la préparation des ébauches d'hameçons (Carlier, 2002 : 80 ). Ce n'est cependant qu'une hypothèse en l'absence de trace visible.

Sans aucune pièce représentative, il est impossible de connaître la forme primaire que prend le support au cours de la première étape d'ébauchage. Cependant, celle-ci évolue assez vite vers une forme plus élaborée tels qu'un pentagone $\left(n^{\circ}\right.$ Atiahara 1407) ou encore un hexagone $\left(n^{\circ}\right.$ Vaito'otia F17-5). A ce stade, la distinction la plus nette est donnée par l'orientation des bords latéraux qui peuvent converger soit vers le bord proximal, soit vers le bord distal, et dont le profil (droit ou concave) introduit également une variété. Il semble que ces formes de support soient préétablies en fonction du système de fixation proximal souhaité. Ainsi, on suppose que les plaquettes hexagonales facilitent l'aménagement ultérieur des ailettes en partie proximale. Notons aussi que les bords distaux sont travaillés de manière à leur donner un profil convexe sur lequel nous reviendrons plus loin.

Durant cette première étape de mise en forme débute également un travail d'abrasion, réalisé en frottant la pièce sur un morceau de corail humidifié. Cette opération a pour double objectif d'enlever le periostracum sur la face externe et d'amincir progressivement le support de nacre en vue d'un façonnage plus élaboré. Bien qu'elle puisse survenir régulièrement au cours du processus de fabrication, l'abrasion commence dès l'ébauchage et avant l'aménagement des parties proximales et distales, comme en témoigne le peigne de Hane ${ }^{\circ}$ E8\#26.

\section{Façonnage des parties distales et proximales}

Une fois le support formé débutent à proprement parler les aménagements des parties proximales, avec la mise en place du système de fixation, et distales, avec le façonnage des pointes. Ici, le schéma opératoire peut suivre deux orientations selon l'ordre d'exécution suivi par l'artisan. Notre corpus montre en effet deux séquences possibles, l'une débutant par la partie distale, l'autre par 


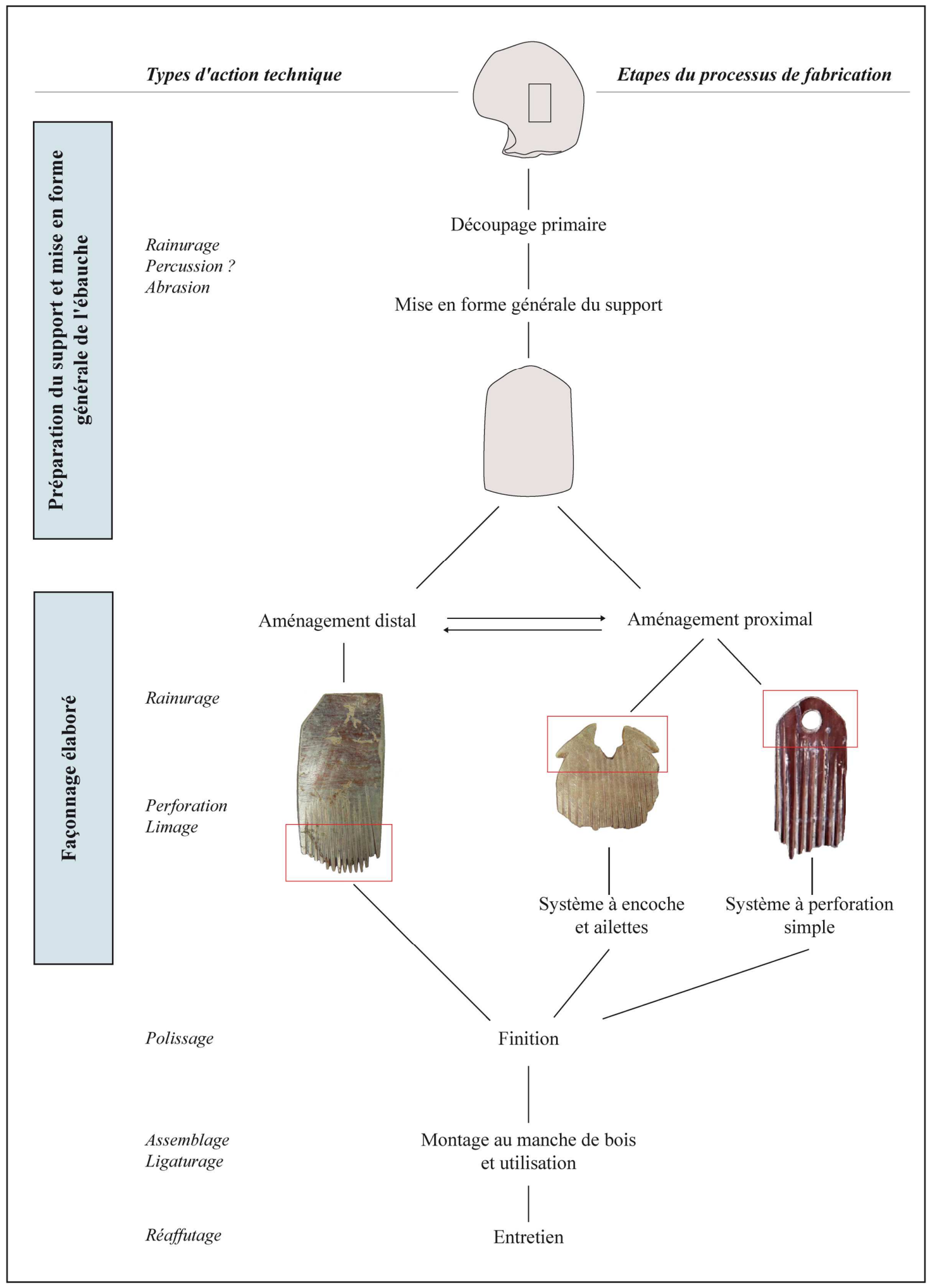

HIGURE 2. - Keprésentation schématique de la chaîne opératoire de tabrication des peignes à tatouer en nacre

la partie proximale. Pour la majorité des objets (cinq pièces sur les huit représentatives), la partie distale a été façonnée en premier. Seules deux pièces, K98-5 et Ô112-11, ont subi un travail de la partie proximale préalablement à la formation des pointes. Ce parti pris de l'artisan ne peut être explicité en raison du faible nombre de pièces composant notre corpus. Il ne semble cependant traduire aucune tradition locale puisqu'il ne s'agit que de deux peignes parmi les neuf découverts 
sur le même site de Hane. Tous sont de même type ce qui implique également que des schémas opératoires différents peuvent être suivis en vue d'aboutir à une même forme. La raison de ce choix serait donc d'ordre pratique, argument qui pourrait être démontré par l'expérimentation.

\section{- Mise en forme des pointes}

L'aménagement de la partie distale consiste à rainurer la matrice nacrée pour en dégager un ensemble de pointes qui constitueront la partie active de l'outil en pénétrant dans la peau du patient lors de l'opération de tatouage.

Comme nous l'avions mentionné précédemment, le bord distal est déjà préparé avant la réalisation des pointes par le façonnage d'un profil convexe, ainsi qu'en témoigne le peigne $n^{\circ}$ O112-11. Cette courbure implique que les extrémités des pointes une fois dégagées ne seront pas toutes sur le même plan. De fait, même avec des pointes d'égales longueurs, celles de la zone centrale du bord convexe seront plus avancées que celles des bords. L'exemplaire $\mathrm{n}^{\circ} \mathrm{N} 88$ 13 de Hane est le seul peigne avec des pointes conservées. Or, on constate là aussi une courbure générale du profil distal à leur extrémité. La différence entre le centre et les bords est de près de $3 \mathrm{~mm}$ pour $\mathrm{O} 112-11$ et $4 \mathrm{~mm}$ pour N88-13. Cette remarque induit une action particulière recherchée sur l'épiderme du patient. En effet, si le peigne est maintenu perpendiculairement à la peau, seules les pointes centrales la pénétreront et non les pointes latérales. Une rotation de la lame du peigne (autour de l'axe du manche) au cours de la percussion est envisageable mais on ignore sa réelle efficacité en l'absence d'observation précise. Une autre explication est donnée par P. et M.-N. Ottino (1998 : 55), selon laquelle cette convexité était donnée aux lames utilisées pour tracer sur la peau des lignes dont la réalisation nécessitait un chevauchement à chaque frappe. Selon cette affirmation, les pointes latérales serviraient de repères pour des impressions suivies. Les deux hypothèses proposées ne s'excluent cependant pas l'une l'autre. Notons que les peignes en os décrits à la période historique ne présentaient pas ce profil convexe, toutes les pointes étant situées sur le même plan.

Le rainurage s'effectue toujours du haut de la plaquette vers le bas, comme en témoignent la largeur et la profondeur progressives des stries en direction du bord distal. La largeur maximale des stries n'excède pas $1 \mathrm{~mm}$. L'opération nécessite donc un outil pointu et très fin tel que des dents animales emmanchées, de rat ou de poisson (de requin notamment), formant une gouge. Souvent, on note que la plaquette de nacre est amincie par abrasion dans la partie distale. Cette opération permet de réduire l'épaisseur de la nacre et ainsi de faciliter la réalisation des pointes.
D'une part, la quantité de matière à rainurer est moindre ce qui réduit l'intensité de la pression exercée sur la gouge et offre un meilleur contrôle du geste. D'autre part, les stries réalisées sur une face apparaissent en négatif sur la face opposée et servent alors de repères pour régulariser le trait lors du rainurage sur l'autre côté. On peut dès lors se demander si les pointes sont dégagées une à une, avec un va-et-vient constant entre les deux faces, ou si une face est d'abord entièrement rainurée. Seul l'exemplaire ${ }^{\circ}$ K98-5 témoignerait de cette seconde proposition, du moins si l'on suppose que les traits en relief sur la face postérieure sont bien des négatifs de rainurage.

Les stries peuvent être plus ou moins longues. Certaines débutent dès la zone proximale et couvrent toute une face ( ${ }^{\circ}$ E9\#58), tandis que d'autres, plus courtes, ne sont réservées quà la zone distale. Le peigne non numéroté de Tubuai présente une face avec des stries longues interrompues par la fracture tandis que l'autre côté montre des stries plus courtes. Cela étant, une même face peut aussi présenter des stries de longueurs variées. Même en l'absence d'expérimentation, on peut supposer qu'un rainurage sur une plus grande longueur offre, d'un point de vue pratique, un meilleur contrôle du geste et du trait.

Nous n'avons pas pu déterminer systématiquement le nombre de pointes des lames étudiées, aussi ne tenons-nous pas compte ci-après des peignes présentant une largeur incomplète et que l'on ne peut objectivement restituer. Le nombre de pointes varie entre neuf $\left(\mathrm{n}^{\circ}\right.$ Atiahara-1407) et 20 ( $n^{\circ} \mathrm{N} 88-13$ et $n^{\circ}$ K98-5). Le

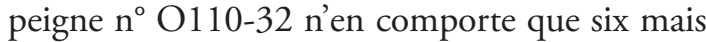
en raison de sa forme particulière, il n'est pas intégré à cet échantillon. Pour trois pièces dont la largeur distale n'est pas conservée, on suppose néanmoins qu'elles présentaient un minimum de 14 pointes. De manière très simple, il apparaît que le nombre de pointes est globalement proportionnel à la largeur de la lame. Si l'on s'attache au rapport entre la largeur distale et le nombre de pointes, on pourrait parler de pièce " équilibrée " pour le peigne d'Atiahara ayant un rapport de 1:1. Le peigne de Hane $n^{\circ}$ E9\#58 présenterait, quant à lui, des pointes serrées avec un rapport inférieur à 1 , alors que celles de Vaito'otia $n^{\circ}$ F17-5 seraient au contraire plus espacées. La pertinence de ce critère est néanmoins très relative.

- Mise en place du système de fixation proximal

Le façonnage élaboré de la partie proximale du support de nacre est lié à un système particulier de fixation du peigne au manche. Deux modes de fixation ont été mis en évidence au sein de notre corpus, impliquant des aménagements différents du sommet des pièces. 


\section{a. Le système à encoche et ailettes}

Nous avons nommé ce mode de fixation "à encoche et ailettes " afin de rendre compte de l'aspect singulier de ces lames en nacre. En effet, aucune source ne décrit ce type d'aménagement pour les peignes en os observés et recueillis à la période européenne. Les pièces présentent trois éléments remarquables: l'encoche centrale que devait traverser le manche, et les deux ailettes latérales servant au bon maintien de la ligature. Ces éléments offrent des variations morphologiques d'une pièce à l'autre.

L'encoche centrale peut être caractérisée par sa forme (quadrangulaire, circulaire ou ovale), son diamètre ou sa largeur, et son degré d'ouverture. Dans la plupart des cas étudiés, l'encoche est réalisée par perforation contre le bord proximal, une technique rendue possible par l'utilisation d'un perçoir à volant dont on sait qu'il était aux périodes anciennes un outil indispensable à bon nombre de productions artisanales. Généralement, les bords sont ensuite repris par limage pour donner à l'encoche sa forme définitive. En revanche, le recours exclusif au limage pour le façonnage de l'encoche n'est pas impossible, à l'instar du peigne $n^{\circ}$ E9\#58 de Hane. Une perforation unique conduit à façonner une encoche étroite (environ 4-6 $\mathrm{mm}$ de diamètre), mais certaines pièces montrent deux ( $c f$. O112-11 et G19-6), voire trois (exemple de Rivnac), perforations juxtaposées chevauchantes et présentent donc des encoches plus larges (de 7 à $9 \mathrm{~mm}$ ). Sur l'objet $n^{\circ}$ E9\#58 et peut-être aussi $n^{\circ}$ P86-27, nous avons également noté la présence d'une encoche plus petite aménagée à partir du bord inférieur de l'encoche principale. Il est fort probable que celle-ci serve, comme les ailettes latérales, au passage et au maintien des ligatures. La même fonction pourrait être attribuée à l'encoche inférieure du façonnage "trilobé " de Rivnac. Enfin le degré d'ouverture doit être pris en compte car il définit aussi la manière de fixer le peigne au manche de bois. Le degré d'ouverture est faible lorsque la largeur de l'espace évidé sur le bord proximal est inférieure au diamètre du bâton. $\mathrm{Ce}$ dernier est alors bien maintenu dans l'encoche et les deux éléments (manche et lame) ne peuvent être dissociés. C'est le cas des pièces G19-6, Rivnac, TP12-4 et O112-11. Au contraire, si le degré d'ouverture est plus élevé, la lame est directement encochée dans le manche mais reste libre en l'absence de ligature (pièces E9\#58 et K985). Le peigne de Hane Surf.\#1 est original car l'encoche centrale présente une forme ovalaire très marquée. On peut envisager l'usage d'un manche en bois dont l'extrémité serait taillée et aplatie pour l'adapter à l'encoche.

Les ailettes latérales sont façonnées ou marquées par des opérations de natures différentes. Les têtes peuvent être dégagées par rainurage lors de la mise en forme initiale de la plaquette. Elles peuvent aussi être aménagées grâce à des gorges transversales limées comme le montre le peigne $\mathrm{n}^{\circ}$ E9\#58. Ces actions contribuent aux morphologies variées qu'affectent les ailettes et que nous résumons dans une typologie de cinq classes ( $c f$. fig. 3) :

- Gorge transversale externe (E9\#58, K98-5)

- Protubérance externe (O112-11)

- Protubérance sommitale (TP12-4)

- Protubérance interne et décrochement latéral (G19-6)

- Protubérance interne et gorge transversale externe (Rivnac)

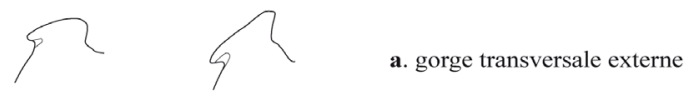

b. protubérance externe

c. protubérance sommitale

d. protubérance interne et décrochement latéral

e. protubérance interne et gorge transversale externe

Figure 3. - Morphologies des ailettes latérales 
a.

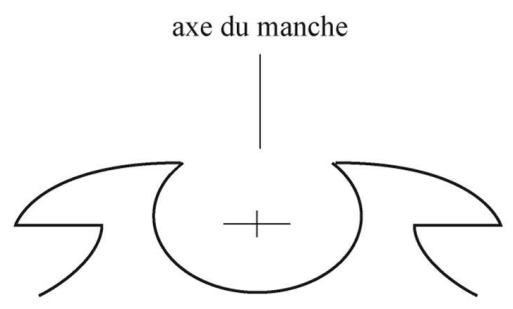

b.

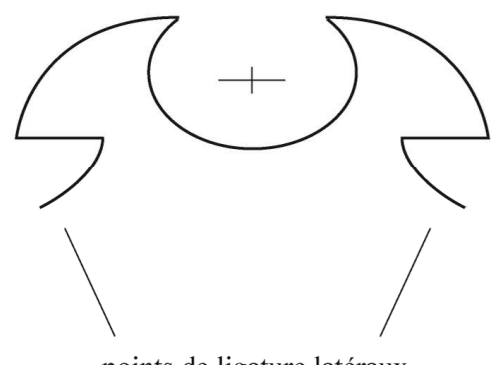

points de ligature latéraux

Figure 4. - Schémas de positionnement des points de ligature latéraux

La mise en forme des types $d$ et $e$ est directement liée au degré d'ouverture de l'encoche dont dépendent les protubérances internes.

Un dernier caractère peut être souligné, celui du positionnement des points de ligature latéraux (gorge, protubérance et décrochement) par rapport à l'encoche centrale. Le plus souvent, ils sont situés plus bas que l'axe du manche mais dans quelques cas (O112-11, TP12-4), ils sont alignés sur un même plan transversal (cf. fig. 4). $\mathrm{Si}$ la forme des ailettes dépend probablement d'une relative volonté esthétique, elle répond sans doute aussi à un besoin pratique qui nous apparaît conditionné par le type de ligature que l'artisan souhaitait employer. Ce mode de fixation unique n'étant pas documenté sur les peignes à tatouer de la période européenne, nous ne disposons d'aucune comparaison explicitant les procédés de ligature. De plus, il n'existe aucune réelle étude à ce sujet si ce n'est pour l'emmanchement des herminettes (Mead, 1968, 1971). De fait, il serait très présomptueux de nous lancer dans une quelconque reconstitution sans même disposer d'un référentiel expérimental exploitant un savoir-faire technique qui, par ailleurs, a presque entièrement disparu aujourd'hui en Polynésie française. C'est donc un pan important du schéma opératoire de fabrication qui nous reste inaccessible en l'état actuel des connaissances.

\section{b. Le système à perforation simple}

Si ce type d'action sur la matière est, semble$\mathrm{t}$-il, commun lors de la préparation des peignes à ailettes, la perforation centrale simple comme mode de fixation à part entière n'a été observée que sur l'exemplaire achevé no 1407 de Atiahara, à Tubuai. Il semble en revanche privilégié pour les peignes en os polynésiens anciens, notamment aux Australes et en Nouvelle-Zélande (Davidson, 1984 : 91).

En raison du très petit diamètre de la perforation $(2,5 \mathrm{~mm})$, on peut s'interroger sur sa fonction. La lame était-elle directement emmanchée ou plutôt attachée ? Très peu d'éléments de comparaisons historiques nous sont parvenus mais la synthèse des quelques informations disponibles, tant dans la littérature que dans les collections muséales, nous permet de proposer trois hypothèses de fixation (fig. 5):

- Emmanchement direct. Des exemplaires sont connus qui montrent à l'extrémité du manche en bois une réduction du diamètre permettant de le faire pénétrer dans la perforation de la lame, ensuite fixée par des ligatures. Ce cas est documenté dans les outliers polynésiens de Tikopia (Pendergrast, 2000) et Ontong Java (Robitaille, 2007: fig.10), mais n'est apparemment pas connu en Polynésie orientale.

- Fixation sommitale. La partie proximale de la lame est posée perpendiculairement au sommet du manche ${ }^{1}$ et maintenue par la ligature. Ce mode de fixation semble propre aux archipels de Polynésie occidentale puisqu'on le retrouve sur les peignes de Tonga et de Samoa (Buck, 1930 ; Galliot, 2011).

- Encochement. L'extrémité proximale de la lame est fixée (soit perpendiculairement soit sous un angle aigu) dans une fente taillée dans la largeur du manche, à proximité de son sommet. Une ligature passant par la perforation centrale permet de la maintenir en place. Ce mode de fixation est assez fréquent en Polynésie orientale puisqu'il est décrit tant aux Marquises (Von den Steinen, 1925) qu’aux îles de la Société (Roth, 1905), aux Cook (Buck, 1927) et en Nouvelle-Zélande (Davidson, 1984). La distribution géographique du système d'encochement pour les peignes en os nous laisse supposer qu'il sagissait également du mode employé pour la pièce de nacre de Atiahara mais n'exclut pas pour autant les autres hypothèses, en particulier le principe de fixation sommitale qui serait tout aussi adapté à la lame de Tubuai au regard de sa forme. Quant à l'exemplaire O110-32, dont la forme est similaire à celles de lames en os connues à la période historique, il était sans aucun doute directement encoché dans le manche. 


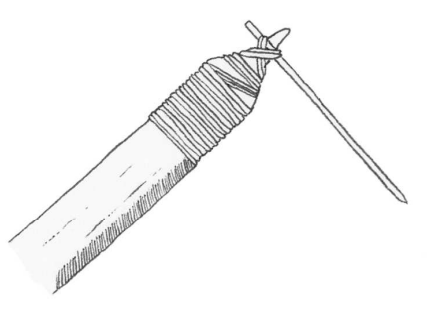

Emmanchement direct

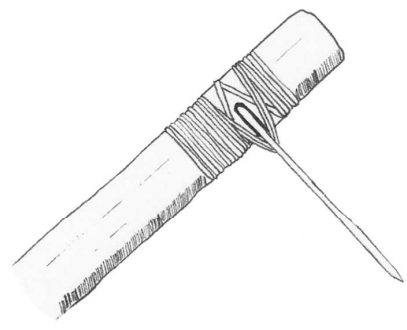

Encochement

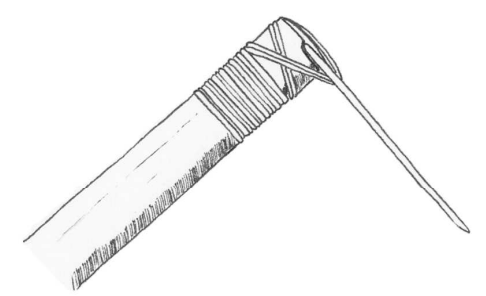

Fixation sommitale

Figure 5. - Reconstitution des trois hypothèses de fixation au manche (dessins G. Molle)

\section{Opérations de finition et d'entretien}

Lorsque la pièce est façonnée, des opérations de finition permettent d'achever la fabrication des lames, notamment un polissage définitif de tous les bords. Bien entendu, la chaine opératoire ne s'arrête pas à ce stade puisque comme n'importe quel outil, les lames de peignes à tatouer devaient être régulièrement entretenues pour leur conserver une efficacité. Les pointes notamment devaient être réaffutées en les frottant sur un support en corail fin ou en peau de requin ${ }^{2}$.Cet entretien n'a cependant pas empêché certaines pièces de se briser par la suite. Au vu des stigmates, il ne fait nul doute que la cassure de l'extrémité des pointes, d'une ailette et même de la pièce complète par fracture au niveau d'une strie, met un terme à l'utilisation de l'objet, sa petite taille empêchant toute intervention de refaçonnage complet. Ce scénario est au moins envisageable pour les pièces G19-6, Rivnac, E9\#58 et Atiahara 1407. Néanmoins, la disparition des pointes suite à des effets taphonomiques n'est pas impossible non plus dans certains cas.

\section{Interprétation typologique et diachronique}

Nulle part ailleurs qu'en Polynésie française, la nacre n'a été utilisée pour la confection des peignes à tatouer. Pour cette raison, ceux-ci n'ont été que très rarement intégrés aux typologies, certes peu nombreuses, de cette catégorie d'objets. Il nous faut souligner le remarquable effort de B. Robitaille (2007) qui pour sa part, a bâti une classification des outils à tatouer emman- chés perpendiculairement ${ }^{3}$. Sa revue exhaustive des connaissances en la matière dans tout le Pacifique s'attachait particulièrement aux objets en os. Toutefois, sa typologie nous permet de mieux distinguer la place des peignes en nacre dans une panoplie océanienne plus générale.

Les peignes en nacre sont ainsi assimilés à la catégorie des lames individuelles qu'il nomme dans son étude "one-piece matrix comb" par opposition aux pièces composites formées de deux ou plusieurs lamelles juxtaposées et/ou superposées (" composite matrix combs"). Ces deux sousgroupes appartiennent à la famille des seuls outils que l'on peut réellement qualifier de peignes et qui ne sont pas des instruments formés de pointes indépendantes liées entre elles ("pointed instruments"). Cette famille de peignes constitue selon lui l'unique groupe monophylétique identifiable en Océanie, représenté majoritairement en Polynésie mais aussi dans quelques régions de Mélanésie et de Micronésie. Les distinctions internes qu'il opère dépendent essentiellement de la largeur des lames. Suivant son propos, toutes les pièces de notre corpus seraient larges sauf celles d'Atiahara-1407 et Hane-O110-32 qui seraient des pièces étroites. Son étude portant sur les peignes en os, il n'est fait aucun usage particulier des aménagements proximaux dans l'élaboration de la typologie, ce qui pose naturellement une certaine limite à son interprétation. À ce titre, nous pensons qu'une réflexion plus systématique sur les modes de fixation serait un moyen utile pour affiner cette première vision générale.

Comme nous l'avons déjà souligné, il n'est fait aucune mention de peignes à tatouer en nacre dans la littérature ethnohistorique. De même, aucun objet de ce type n'a, à notre connaissance,

2. On peut envisager qu'en cas de bris d'une ou plusieurs pointes, l'artisan souhaitant encore exploiter le peigne abrase la partie distale avant de retailler de nouvelles pointes en réutilisant les stries de rainurage précédentes.

3. Un type d'outils qu'il nomme « Perpendicularly-hafted tattooing instruments » ou PHTI et qui est une particularité austronésienne. Les deux autres variantes sont les instruments dont la pointe est alignée avec le manche (《straight-hafted ») et les aiguilles utilisées dans le procédé de « couture » (« needle and thread ») propre aux cultures sibériennes. 
été collecté par les voyageurs européens suite au Contact ${ }^{4}$. À cette époque, toutes les descriptions s'accordent à dire que les matériaux privilégiés pour la fabrication de ces outils particuliers étaient l'os, surtout de chien ou d'oiseaux voire humains, ou l'écaille de tortue (Ottino, 1998 : 53). Il existait aussi des pointes constituées à partir de dents de requin ou de barracuda servant de poinçons de tatouage aux Tuamotu et peut-être aux Gambier (Emory, 1975 : 97 ; Buck, 1938 : 179). L'emploi de ces matériaux est commun à tous les archipels de Polynésie orientale, même si des formes d'outils diverses existaient à Hawaii, en Polynésie centrale et en Nouvelle-Zélande.

Est-ce à dire que l'usage de la nacre avait été abandonné et n'était déjà plus réservé à cette époque qu'à la fabrication d'outils usuels tels que les hameçons? Si oui, peut-on le confirmer au travers des contextes chrono-stratigraphiques? La résolution de ces derniers n'est pas toujours suffisante pour avancer une réflexion pertinente, car nous sommes tributaires à la fois de la qualité des enregistrements stratigraphiques et des datations des couches archéologiques.

Les trois peignes de Hane découverts en 2009 proviennent tous de la couche G, c'est-à-dire un niveau de pavage d'habitat dont la mise en place a été datée autour de 1200 A.D. (Molle, 2011 : 136). Les contextes de découverte des peignes recueillis par Sinoto sont beaucoup moins précis en l'absence de toute publication détaillée. Trois d'entre eux (O110-32, P108-5 et O112-11) proviennent du niveau III de l'aire A dont on a démontré ailleurs que la séquence chronologique ne pouvait être affirmée en l'état (op.cit. : 130). Les autres peignes ont été découverts dans l'aire $\mathrm{B}$, dans les niveaux $\mathrm{V}$ et VI qui correspondent à la fin de la première phase d'occupation du site dunaire et la construction des pavages, soit une date contemporaine de celle indiquée plus haut. L'exemplaire recueilli en surface est plus problématique mais il provient d'un secteur de la zone côtière ayant semble-t-il connu des remaniements récents.

Le peigne de Ha'atuatua à Nuku Hiva fut découvert dans la couche $\mathrm{C}$ du sondage TP11-14, datée entre 1275 et 1475 A.D. (Rolett et Conte, 1995 : 222).

Les deux exemplaires de Vaito'otia proviennent du niveau IV, initialement estimé à 1180 \pm 90 A.D. (Sinoto, $1983: 594$ ) mais pour lequel une datation récente semble indiquer une occupation entre 1100 et 1200 A.D. (A. Anderson, com. pers., août 2009).
Le peigne du site de Rivnac fut découvert dans la couche 3 de la zone 2 supposée ancienne mais pour laquelle on ne dispose à l'heure actuelle d'aucune datation. Une réappréciation du site par T. Maric est en cours, qui devrait apporter de nouveaux éléments de chronologie.

Concernant les exemplaires de Tubuai, seule la pièce $n^{\circ} 1407$ fut recueillie en contexte au cours de la fouille du site de Atiahara. Elle fut, semble-t-il, découverte à l'interface des niveaux D/E (L. Miller et A. Hermann, com. pers., mai 2012), avec plusieurs peignes en os. Il n'existe aucun rapport ou document publié sur les résultats de cette campagne hormis un article récent dans lequel sont indiquées deux dates de $930 \pm 60$ B.P. et $855 \pm 30$ B.P. (soit entre les $\mathrm{XI}^{\mathrm{e}}$ et $\mathrm{XIII}^{\mathrm{e}}$ siècles de notre ère) se rapportant à des couches qui ne sont pas clairement identifiées mais dont on suppose qu'elles marqueraient un terminus post-quem (Worthy et Bollt, 2011 : 73). Les deux autres pièces décrites pour Tubuai ont été découvertes en surface, dans les zones de labour à proximité de Atiahara.

La revue rapide des contextes chronologiques, parfois très incertains, semble malgré tout indiquer que la majorité des peignes en nacre remonte à des périodes assez anciennes comprises entre les $\mathrm{XI}^{\mathrm{e}}$ et $\mathrm{XV}^{\mathrm{e}}$ siècles, soit bien avant l'arrivée des premiers Occidentaux en Polynésie orientale. Sans remonter toute la séquence océanienne proposée par Robitaille dans son étude, nous ne pouvons cependant qu'accorder du crédit à son scénario de développement technologique des instruments polynésiens. Faisant suite à l'invention ou à l'introduction des peignes (matrix combs), plutôt étroits, en Polynésie occidentale, la panoplie d'outils s'enrichit de pièces plus larges destinées notamment à la réalisation d'aplats, ce qui sous-tend aussi une évolution des motifs et de la manière de les réaliser. Robitaille avançait ainsi l'idée selon laquelle une période de changements culturels, sociaux et esthétiques s'ouvrait avec l'installation des communautés en Polynésie orientale, justifiant l'apparition de peignes larges dans les assemblages archéologiques anciens. Le développement conjoint de pièces larges et étroites réalisées tant dans de l'os que dans de la nacre est en effet attesté comme en témoignent les collections découvertes dans les archipels centraux, mais aussi à Mangaia aux Cook (Kirch $e t$ al., 1995) et en Nouvelle-Zélande (Davidson, 1984). La réalisation de peignes larges a pu cependant se poser comme une véritable difficulté, un élargissement des lames allant de pair avec

4. Nous n'avons trouvé qu'une seule référence d'un objet en nacre décrit comme un peigne à tatouer. Il s'agit d'une pièce conservée au musée du quai Branly à Paris et enregistrée dans l'inventaire sous le nº 71.1880.49.29. Sa nomenclature indique qu'elle provient des îles Salomon, sans plus de précision. N'ayant pu étudier cette pièce plus en détail, nous ne pouvons pas nous avancer sur sa véritable fonction. Néanmoins, les dents ou pointes visibles ne sont pas réalisées par la même technique de rainurage. 

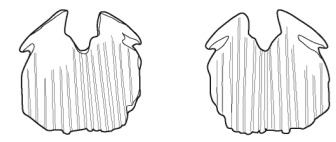

E9 \#58
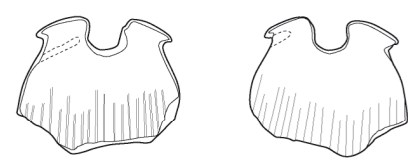

K98-5

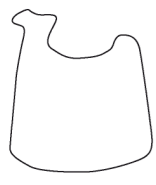

O112-11

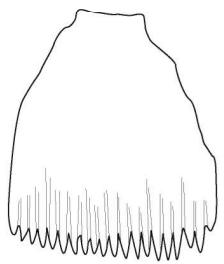

N88-13

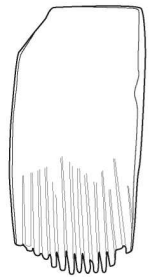

E8 \#26

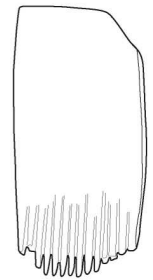

P86-27

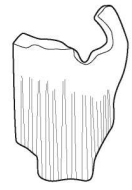

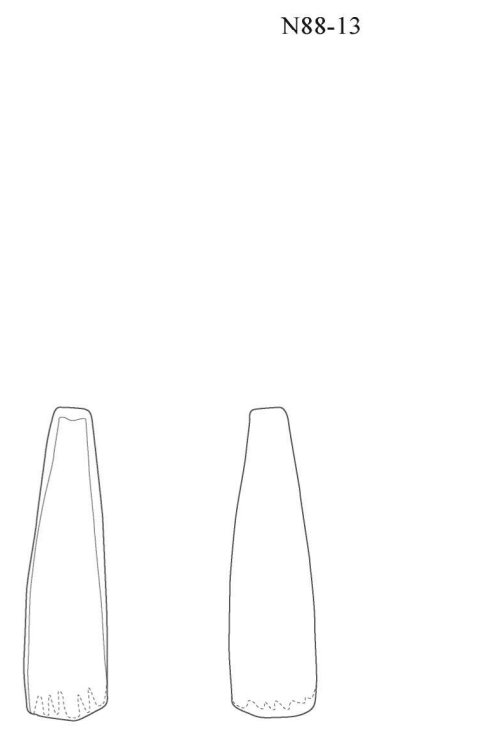

0110-32
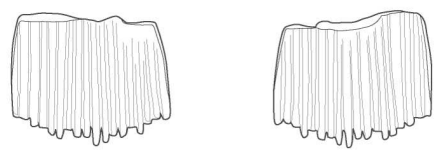

F7 \#34

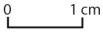

Figure 6. - Peignes à tatouer découverts sur le site de Hane, Ua Huka (Marquises)

une plus grande fragilité qui provoque souvent des cassures sous l'effet répété de la percussion. D’après notre corpus, deux voies d'expérimentation technique ont pu être suivies : d'une part, un travail important sur la partie proximale et le mode de fixation par ailettes qu'on retrouve aux Marquises et aux îles de la Société ; d'autre part, un accroissement de la résistance par un épaississement des lames en os comme cela semble être le cas aux Australes avec les assemblages de Atiahara ainsi qu'en Nouvelle-Zélande avec les peignes en os de moa. Dans ces deux derniers cas, le mode de fixation par encochement ou fixation sommitale reste comme auparavant 
usité, montrant peut-être la permanence d'une tendance culturelle ancienne.

Néanmoins, aux environs du $\mathrm{Xv}^{\mathrm{e}}$ siècle, l'usage de la nacre est abandonné de même que le système d'ailettes. Les artisans privilégient dès lors d'autres matériaux dont l'os, en plus grande variété, et l'écaille de tortue, en particulier aux Marquises (Ottino, 1998 : 53) et probablement aux Tuamotu ${ }^{5}$, et inventent de nouvelles formes de peignes. L'apparition des pièces composites aux îles de la Société et à Hawaii (ainsi qu’à Samoa où les pièces sont plus complexes encore) constitue sans doute l'exemple le plus significatif puisque ces dernières permettent de réduire l'effet de fracture par la juxtaposition de plusieurs lames étroites et indépendamment moins fragiles (Robitaille, 2007: 168). Les fixations par encochement sont dès lors les seules employées. Les composites continueront d'être utilisés dans la panoplie des tatoueurs avec les peignes étroits bien après l'arrivée des Occidentaux. L'absence de peigne composite aux Australes et en Nouvelle-Zélande semble confirmer dans ces régions une permanence des formes larges anciennes, un argument qui pourrait aussi être justifié par la découverte de peignes en nacre datant de périodes plus récentes à Tubuai ( $c f$. Ambrose, $2012: 7)$.

Se dessine ainsi un scénario plausible de développement des instruments de tatouage qui témoigne à la fois de la capacité d'adaptation dont firent preuve les artisans et d'une évolution culturelle par laquelle des tendances régionales apparaissent comme de nouveaux marqueurs à prendre en compte dans des réflexions générales sur les séquences polynésiennes.

\section{Conclusion}

Notre étude d'un corpus le plus exhaustif possible de peignes en nacre découverts dans les archipels centraux de Polynésie a démontré l'utilité d'une analyse technologique des schémas opératoires dans la reconnaissance des choix réalisés par les fabricants. La mise en relief de leurs caractéristiques originales au sein d'une réflexion typologique nous a permis de confirmer l'hypothèse d'un scénario de développement régional. Des études de ce type portant sur des collections plus complètes regroupant des objets en matériaux divers seraient profitables. Ayant pressenti l'existence de tendances culturelles marquées, nous pensons par ailleurs qu'une attention parti- culière doit être portée aux modes de fixation et aux aménagements sommitaux des peignes.

Le schéma opératoire décrit dans cet article s'avère finalement complexe à mettre en œuvre et, au-delà du questionnement technique, renvoie à l'identité et au statut de l'artisan qui doit en maîtriser tous les aspects. Le tatoueur aux périodes anciennes ${ }^{6}$ fabriquait-il lui-même ses propres outils ou faisait-il appel à un spécialiste du travail de la nacre? Quelques remarques iraient dans le sens de la première proposition. D'une part, il ne fait aucun doute que la forme des peignes, leur largeur notamment, est adaptée aux besoins spécifiques du tatouage et des motifs. Or, seule la connaissance et la maitrise du geste lors de l'opération favorisent l'adaptation technique. De manière plus pragmatique, il est tout aussi évident que le tatoueur est lui-même en charge de l'entretien de ses lames, ainsi que de leur montage et démontage impliquant un savoir-faire quant aux ligatures. Enfin, rappelons que le tatouage est un acte très important et de ce fait, requiert des outils dont la fabrication et l'usage étaient entourés de précautions et de tapu. Ces quelques remarques, malheureusement fort peu étayées par les commentaires ethnographiques tardifs, tendent à placer l'ensemble de la chaîne opératoire sous la direction d'un seul et même individu qui s'affirme ainsi dans l'ancienne société polynésienne comme un spécialiste, tant dans le domaine technique de fabrication des peignes que dans celui du tatouage à proprement parler, et de la conduite des rituels et le respect des tapu entourant cette opération chargée de signification.

\section{ANNEXE : DESCRIPTION DES PEIGNES À TATOUER EN NACRE}

Hane (Ua Huka, Marquises), n E9 \#58 (fig. 6)

Cette pièce achevée est presque entière. La plaquette de nacre de forme carrée $(16 \mathrm{~mm}$ de longueur pour $15 \mathrm{~mm}$ de largeur maximale) présente une épaisseur de $1 \mathrm{~mm}$ égale sur toute la surface de la pièce, hormis à l'extrémité distale qui est amincie. La pièce a été découpée par rainurage bifacial, sans doute dans la partie centrale de la valve, mais il est impossible de distinguer les faces externe et interne en raison du procédé d'abrasion qui a fait disparaître toute trace de periostracum. Les bords sont parallèles sauf en par-

5. Les motifs de tatouage aux Tuamotu et aux Gambier, documentés par la littérature ethnohistorique, se caractérisent par de grands aplats ce qui implique l'usage de peignes larges. Néanmoins, il n'est fait aucune référence à ce type de peigne dans la bibliographie spécialisée sur ces archipels.

6. À la période européenne, du moins aux Marquises, il ne fait aucun doute que le tatoueur, aidé de son assistant, fabriquait lui-même ses peignes (Ottino, 1998 : 52). 
tie proximale où ils convergent vers le sommet aménagé de façon à fixer le peigne au manche.

Les deux surfaces de la pièce sont parcourues par des stries de rainurage ayant permis la réalisation des pointes. Il ne fait aucun doute qu'elles ont été réalisées dans un premier temps puisqu'elles sont interrompues par l'encoche sommitale et les échancrures des ailettes latérales et ce sur les deux faces. Elles sont au nombre de 15 et forment ainsi 16 pointes dont il manque les extrémités brisées. À l'origine, le peigne devait toutefois présenter 17 pointes. En effet, le bord droit est, semble-t-il, cassé au niveau d'une rainure ce qui a provoqué un enlèvement de matière. En tenant compte d'une symétrie initiale de l'objet, nous pouvons rétablir sa largeur réelle et affirmer qu'une pointe a entièrement disparu.

L'aménagement de la partie proximale du peigne s'est déroulé dans un second temps. Il s'agit d'une encoche centrale de 4 × $3 \mathrm{~mm}$ au sommet de la pièce. Les bords ont été limés pour leur donner un profil rectiligne. On note aussi le dégagement volontaire d'une petite partie $(2 \times 1 \mathrm{~mm})$ au bord circulaire immédiatement sous l'encoche principale. De part et d'autre, des gorges d'environ $1 \mathrm{~mm}$ de profondeur forment les ailettes latérales. Les traces de limage sont bien visibles et recouvrent les stries de rainurage. Elles sont cependant moins marquées, voire à peine amorcées, sur la face interne où les échancrures sont d'ailleurs moins symétriques que sur la face opposée.

\section{Hane (Ua Huka, Marquises), n K98-5 (fig. 6)}

Cette pièce mesure $19 \mathrm{~mm}$ de longueur pour $22 \mathrm{~mm}$ de largeur maximale. Son épaisseur de $2 \mathrm{~mm}$ est régulière. L'une des faces, plus mate que l'autre, montre des trainées d'abrasion ce qui laisse supposer qu'il s'agit de la face externe de la valve huîtrière.

L'aménagement de la partie proximale est achevé et tous les bords ont été limés et même polis. L'encoche principale présente une forme circulaire. Les échancrures latérales, d'une profondeur de $1 \mathrm{~mm}$, sont nettes. Une seule trace très légère de limage est visible sur la face externe. Il s'agit là d'une opération ayant pu servir à mettre en forme l'ailette mais son absence sur les autres bords indique qu'elle n'était peut-être pas nécessaire dans la mesure où les échancrures étaient préalablement bien marquées. Une autre trace de limage apparaît sur la face interne mais celle-ci est accidentelle car située bien plus bas que l'échancrure.

La partie distale est incomplète en raison d'une ou plusieurs fractures ayant provoqué la disparition des pointes sur toute la largeur du peigne. On compte 19 stries de rainurage sur la face interne soit 20 pointes. Les stries étant interrompues par la ligne de fracture, l'opération de rai- nurage sur cette face était achevée avant que la pièce ne soit brisée. En revanche, la face opposée montre des traces de nature différente qui soulèvent plusieurs questions. En effet, il ne s'agit pas ici de stries à proprement parler mais de très fines lignes marquées en relief. On pourrait supposer que cet aspect particulier est donné par le rainurage plus ou moins fortement appuyé sur l'autre face et qui « imprime » en négatif sa marque sur celle-ci. Cette hypothèse nous semble plausible en raison de la structure de la nacre. Rappelons qu'elle est faite de la superposition de fines lamelles de carbonate de calcium qui en font un matériau résistant mais également sensible aux forces exercées à sa surface. Il est donc envisageable que «l'écrasement " des lamelles provoqué par le rainurage aboutisse à une impression négative en relief de l'autre côté. Seule l'expérimentation pourrait valider cette proposition. Concernant la pièce K98-5, l'artisan aurait d'abord réalisé le rainurage d'une face avant de faire de même sur l'autre, mais la cassure survenant peut-être à ce moment-là aurait entraîné son abandon, ce qui explique que la seconde face ne soit pas achevée. Une telle assertion implique aussi que le rainurage est survenu après l'aménagement de la tête.

\section{Hane (Ua Huka, Marquises), n E8 \#26 (fig. 6)}

Cette ébauche a été abandonnée en cours de fabrication. Il s'agit d'une plaquette de nacre rectangulaire de $31 \mathrm{~mm}$ de longueur, $17 \mathrm{~mm}$ de large et $2 \mathrm{~mm}$ d'épaisseur. C'est l'un des plus grands modules du corpus. Les deux faces montrent des traces d'usure traduisant un travail d'amincissement de la plaquette mais l'une d'entre elle, plus sombre, signale la face externe.

Lopération de rainurage est bien visible alors que la tête ne présente aucun aménagement. La réalisation des pointes est donc survenue dans un premier temps. Les stries de rainurage sont surtout présentes dans le tiers distal de la pièce, par ailleurs plus fin, et ne remontent pas aussi haut que sur l'exemplaire E9\#58.

Sur la face externe, on compte 14 stries de rainurage dont l'une est recoupée par le bord droit, tandis que seules 13 stries sont visibles sur l'autre face. En revanche, ce sont bien 14 pointes que l'on observe sur la pièce. Le bord gauche montre, quant à lui, des traces de découpage bifacial et de reprise, indiquant qu'il s'agissait bien du bord de l'objet lui-même. Â l'opposé, on suppose que le bord droit s'est cassé de manière longitudinale après le rainurage, puis a été repris pour ne pas abandonner la pièce mais en produire une de moindre largeur (il est toutefois difficile d'estimer la largeur initiale de l'objet). Notons que sept extrémités de pointes sont bien conservées et montrent une longueur moyenne de $3 \mathrm{~mm}$ pour la partie active. 
Dans la partie proximale, l'angle gauche présente lui aussi une fracture plus nette qui n'a pas été reprise. On peut penser que celle-ci est intervenue en dernier lieu provoquant l'abandon définitif de la pièce pour laquelle l'aménagement de la tête s'avérait impossible en l'état.

\section{Hane (Ua Huka, Marquises), $n^{\circ}$ F7 \#34 (fig. 6)}

Cette pièce étant cassée, on ignore si elle était achevée ou non. Nous ne disposons que de la partie distale, occupée par les rainures. L'objet mesure $12 \mathrm{~mm}$ de long et $20 \mathrm{~mm}$ de large pour une épaisseur de $1 \mathrm{~mm}$. Les deux bords sont légèrement convergents vers la partie proximale. Bien qu'un peu émoussés, ils montrent des traces d'un découpage bifacial ensuite repris par polissage. La largeur de la pièce est donc bien celle initiale de l'objet. Les pointes sont achevées en partie distale. Les stries de rainurage visibles sur les deux faces sont interrompues par une cassure transversale qui a sans aucun doute entraîné l'abandon de la pièce. Elles débutaient plus haut vers le sommet. On en compte 18 qui forment 19 pointes dont seules deux sont bien conservées ( $2 \mathrm{~mm}$ de longueur maximale). Les stries latérales sont, quant à elles, interrompues par le limage et le polissage des bords, ce qui démontre que cette opération est survenue après l'aménagement des pointes.

\section{Hane (Ua Huka, Marquises), n P108-5 (fig. 6)}

Il s'agit d'une pièce incomplète très probablement cassée en cours de fabrication. Le support de nacre mesure $26 \mathrm{~mm}$ de longueur pour 9 $\mathrm{mm}$ de largeur et $1,5 \mathrm{~mm}$ d'épaisseur. Les parties proximales et distales sont aménagées à des stades différents. L'opération de mise en forme des pointes distales était, semble-t-il, achevée. En effet, on note sur ce fragment conservé sept stries sur les deux faces pour huit pointes encore visibles. Leur extrémité est brisée mais sur celles partiellement préservées, les pointes sont dégagées d'environ $1 \mathrm{~mm}$, ce qui suppose que la hauteur de la pièce est complète. La majorité des stries de rainurage débute dans le tiers distal de l'objet même si deux d'entre elles sont plus longues.

Dans la partie proximale, on note une rupture $\mathrm{du}$ bord droit qui converge très nettement vers le sommet. De plus, on constate une trace de perforation biconique au centre de la tête. Celleci avait probablement pour but d'aménager soit une perforation centrale, soit une encoche comme sur les exemplaires E9\#58 et K98\#5 décrits plus haut. Cependant, il apparaît que l'opération s'est soldée par une cassure longitudinale très nette qui a suivi la huitième strie dans la partie distale. Sur cet objet comme sur d'autres, les stries apparaissent comme un point de frac- ture privilégié en raison de leur plus grande fragilité. De fait, toute la moitié gauche du peigne est manquante.

Selon la manière dont on envisage le système d'attache proximale, l'estimation de la largeur initiale varie. Si l'on considère qu'il s'agit d'un mode de fixation par encoche et ailettes latérales (qui dans ce cas n'auraient pas encore été travaillées), alors la perforation correspondrait à une première étape suivie d'un travail de régularisation par limage. En nous basant sur la forme des pièces précédentes, on peut supposer une largeur initiale de $18-20 \mathrm{~mm}$. S'il s'agit d'une perforation centrale simple, alors cette largeur serait réduite à $15-16 \mathrm{~mm}$ environ. Il est néanmoins difficile de trancher sur la question du mode de fixation.

\section{Hane (Ua Huka, Marquises), n P86-27 (fig. 6)}

Cette pièce est incomplète en raison de plusieurs fractures tant en partie proximale que distale. Il s'agit d'un support de nacre de $21 \mathrm{~mm}$ de longueur, $14 \mathrm{~mm}$ de largeur et 1,5 mm d'épaisseur. Le bord gauche encore visible est celui de la pièce elle-même car les bords sont limés et polis. De fait, on peut considérer que la largeur indiquée ci-dessus, prise dans la partie haute du peigne correspond à la largeur initiale voulue par l'artisan. En revanche, il est difficile d'estimer la hauteur initiale de l'objet en raison de la cassure en partie distale. Il semble que cette dernière ait été aménagée par 12 stries de rainurage pour au moins 13 pointes. Si les cassures ont provoqué un enlèvement transversal, on note aussi que le bord inférieur droit s'est détaché en suivant, comme sur d'autres exemplaires, l'une des stries. Le peigne comportait donc originellement plus de 13 pointes. Les stries sont assez longues et occupent les deux-tiers de la lame.

La tête présente des traces d'aménagement qu'il n'est pas aisé de définir précisément dans la mesure où nos observations n'ont été réalisées qu'à partir de photographies. On note sur le bord supérieur droit une protubérance interne de $5 \mathrm{~mm}$ de long. Celle-ci semble avoir été formée par une perforation en partie centrale. Une sorte de petite encoche est également visible sur le bord inférieur de la perforation. Partant de cette encoche, une ligne de fracture court vers le bord gauche et a provoqué un enlèvement de matière important, dont sans doute l'ailette opposée. La question se pose de savoir à quel moment s'est produite la fracture. On suppose que la reprise de la perforation et/ou de l'encoche inférieure a conduit à la cassure. Cette assertion impliquerait qu'il s'agit d'un mode de fixation par encoche et ailettes. L'aménagement de ces dernières ne semble pas avoir été entrepris, comme sur la pièce P108-5. En revanche, l'encoche, s'il s'agit bien de cela, ne semble pas être placée en posi- 
tion centrale. Il n'est guère possible de trancher entre la possibilité d'une « erreur » de l'artisan et celle d'une forme de fixation au moyen d'une encoche plus large que celles observées sur d'autres pièces. La fracture est survenue soit en même temps, soit après l'aménagement des pointes car si la cassure distale était survenue auparavant, et dans la mesure où celle-ci est bien trop préjudiciable à l'emploi du peigne, l'objet aurait été abandonné immédiatement et l'aménagement du sommet aurait été inutile.

\section{Hane (Ua Huka, Marquises), $n^{\circ}$ N88-13 (fig. 6)}

Ce peigne constitue l'un des plus gros modules de notre corpus. Il mesure $32 \mathrm{~mm}$ de longueur pour une largeur maximale de $25 \mathrm{~mm}$ et une épaisseur de 1,5 $\mathrm{mm}$. C'est le seul exemplaire présentant ses pointes en bon état de conservation (une seule est brisée). On compte 19 stries de rainurage pour 20 pointes (détachées de 3 $\mathrm{mm}$ en moyenne). Les stries occupent surtout le tiers distal de la pièce et sont donc assez courtes. Notre observation à partir de la photographie fournie par Y. Sinoto nous indique que les bords latéraux ne sont pas entièrement conservés. Des fractures sont survenues sur les deux bords du peigne ainsi que dans sa partie proximale. De ce fait, aucune trace d'aménagement du sommet n'est visible et il est impossible de reconstituer le mode de fixation.

\section{Hane (Ua Huka, Marquises), nº112-11 (fig. 6)}

Cette ébauche a été abandonnée en cours de fabrication. Le support de nacre de forme globalement trapézoïdale mesure $20 \mathrm{~mm}$ de longueur, $18 \mathrm{~mm}$ de largeur et $1,5 \mathrm{~mm}$ d'épaisseur. Il a été découpé par rainurage mais on ne peut affirmer à partir de la photographie dont nous disposons si les bords ont été repris. Les deux bords latéraux sont convergents vers le sommet. Il est intéressant de noter qu'avant même le rainurage des pointes, la bordure distale a été préparée selon une courbure convexe. Aucune strie n'est visible alors qu'un travail important a été réalisé sur la partie proximale. C'est la seconde pièce du corpus (avec K98-5) à présenter un schéma opératoire dans lequel l'aménagement du sommet précède celui des pointes. En effet, on note que l'extrémité gauche est formée par une protubérance externe très marquée d'environ $2 \mathrm{~mm}$. Cette ailette a fait l'objet d'un travail fin qui lui donne une forme relativement élaborée. L'encoche centrale est plus large que sur d'autres exemplaires. Il est fort probable que sa préparation ait nécessité plusieurs perforations successives dont les bords ont ensuite été soigneusement limés. Néanmoins, l'ailette latérale droite est cassée. S'agit-il d'une fracture survenue au cours de la finition de l'encoche? Quoi qu'il en soit, la disparition de cet élément essentiel a provoqué l'abandon de l'objet avant même que ne débute la réalisation des pointes.

Hane (Ua Huka, Marquises), nº110-32 (fig. 6)

Cette pièce apparemment non achevée est très différente des autres peignes en nacre. Il s'agit d'un support de forme allongée, mesurant 40 $\mathrm{mm}$ de long. Sa largeur varie entre $5 \mathrm{~mm}$ à l'extrémité proximale et $11 \mathrm{~mm}$ à l'extrémité distale. Il a été découpé dans une partie épaisse de la valve, à proximité de la charnière. Son épaisseur est de 3-4 $\mathrm{mm}$, formant au sommet une section rectangulaire. La face externe du peigne est identifiable par le periostracum conservé. Les bords convergents vers le sommet sont émoussés mais montrent bien des traces de reprise par limage et polissage. Des stries de rainurage sont visibles sur l'extrémité proximale. Elles sont très courtes (3 $\mathrm{mm}$ au maximum) et peu marquées. Aussi, aucune pointe n'est encore dégagée en raison sans doute de l'épaisseur de cette zone. On compte cinq stries ce qui suppose que six pointes devaient être formées. L'aspect général de la pièce, très allongé et étroit, la distingue du reste de la collection. Elle se rapproche cependant de la forme qu'adopteront les peignes en os décrits à la période européenne.

\section{Hane (Ua Huka, Marquises), n Surf.1 (fig. 7)}

Cette pièce fut découverte hors contexte archéologique. Elle est incomplète en raison de plusieurs fractures survenues en parties distale et proximale. En revanche, les deux bords sont conservés. Le peigne mesure $16 \mathrm{~mm}$ de long et $18 \mathrm{~mm}$ de large. Les deux faces sont très délitées et il n'est pas possible d'établir l'épaisseur initiale du support de nacre.

Les stries de rainurage, au nombre de 15 , sont visibles. Elles formaient donc 16 pointes à l'origine. Leur extrémité est manquante en raison de la fracture distale. La partie proximale présente des aménagements avec l'ailette gauche marquée par une protubérance interne et une gorge transversale externe. La longueur de l'ailette est de $7 \mathrm{~mm}$. L'ailette droite est désormais cassée mais on peut toutefois reconstituer la forme générale de l'encoche centrale. Celle-ci dessine un ovale large d'environ $8 \mathrm{~mm}$ et haut de près de $3 \mathrm{~mm}$.

Ha'atuatua (Nuku Hiva, Marquises), $n^{\circ}$ TP12-4 (fig. 8)

Ce peigne achevé mesure $23 \mathrm{~mm}$ de long et $16 \mathrm{~mm}$ de large. Les bords latéraux sont légèrement convergents vers le sommet. Bien que nous ne disposions que d'une photographie, il 

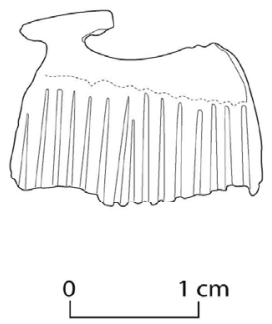
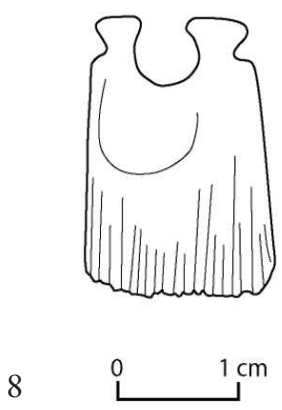

Figures 7-8. - Peignes à tatouer (Marquises).

7. $n^{\circ}$ Surf.\#1 découvert hors contexte à Hane, Ua Huka

8. découvert sur le site de Ha’atuatua, Nuku Hiva

semble bien que tous les bords aient été limés et polis après le découpage par rainurage bifacial. La face antérieure représentée sur la figure 7 correspond à la face interne de la valve car on distingue nettement à la surface de la nacre la zone creuse permettant l'attache du muscle adducteur. En partie distale, on compte 20 stries pour 21 pointes, mais l'extrémité de celles-ci est désormais brisée. La majorité des stries est réalisée à partir du quart distal mais quelques-unes débutent plus haut vers le milieu de la plaquette.

La partie proximale a fait l'objet d'un travail particulier. On retrouve ici le principe de fixation par encoche centrale et ailettes latérales. L'encoche de $6 \mathrm{~mm}$ de diamètre est réalisée par une ou plusieurs perforations dont les bords ont ensuite été repris pour les régulariser. De part et d'autre de l'encoche sont formées des ailettes également marquées par des décrochements assez ouverts des bords latéraux $(1 \mathrm{~mm}$ de profondeur). Il ne s'agit pas à proprement parler d'ailettes latérales car les deux têtes sont orientées vers le haut. Notons une légère dissymétrie, l'encoche étant décentrée vers le bord gauche et l'ailette droite étant plus large.

Vaito'otia (Huahine, Iles-Sous-le-Vent), $n^{\circ}$ G196(fig. 9)

Ce peigne mesure $19 \mathrm{~mm}$ de long, $21 \mathrm{~mm}$ de large et $2 \mathrm{~mm}$ d'épaisseur maximale. Il est complet à l'exception des extrémités des pointes dont la plupart sont brisées. Les bords latéraux sont parallèles. En raison de la faible résolution de la photographie dont nous disposons, il est difficile de compter avec certitude le nombre de stries et de pointes. Il semble que les stries soient assez courtes et réservées à la partie distale. Il est surprenant de constater des enlèvements de formes identiques aux deux angles distaux, formant ainsi un rétrécissement de la largeur en direction de la partie active comportant les pointes. Il n'est pas aisé de dire s'il s'agit d'un aménagement vo- lontaire ou non ; mais si tel était le cas, ce serait un fait unique dans notre corpus puisquailleurs, les pointes débutent dès le bord des pièces.

Le sommet du peigne montre un système de fixation par encoche et ailettes. Ces dernières sont formées de deux protubérances internes marquées notamment par un décrochement des bords latéraux. L’encoche centrale a été réalisée par deux perforations dont on observe encore la jonction sur la bordure inférieure. Les bords ont ensuite été régularisés.

Vaito'otia (Huahine, Îles-Sous-le-Vent), $n^{\circ}$ F17-5 (fig. 9)

Ce support de nacre prend la forme d'un hexagone allongé de $22 \mathrm{~mm}$ de longueur pour $19 \mathrm{~mm}$ de largeur maximale. Les deux bords latéraux principaux sont convergents vers la partie distale occupée par les stries de rainurage au nombre de 10, marquant 11 pointes désormais brisées. Les stries sont assez courtes sur les côtés et plus longues au centre de la plaquette sans toutefois dépasser la moitié de la pièce. La tête n'est pas aménagée si ce n'est par une rupture des bords principaux dont le quart supérieur converge vers la bordure sommitale. On n'observe pas de trace de perforation ou de limage, mais la pièce n'est aucunement cassée. L'objet semble donc avoir été abandonné en cours de fabrication pour une raison inconnue. En l'absence d'aménagement proximal, on ne peut restituer avec certitude le mode de fixation prévu à l'origine mais le système d'ailettes semble le plus probable au vu de la largeur de la pièce.

\section{Rivnac (Tahiti, Illes-du-Vent), s. $n^{\circ}$ d'inventaire (fig. 10)}

Ce peigne est constitué d'un support de $21 \mathrm{~mm}$ de long, $18 \mathrm{~mm}$ de large et épais de $1 \mathrm{~mm}$. Les bords latéraux sont parallèles sauf en partie distale où le bord gauche s'oriente vers le bas de la pièce. On compte 16 stries sur la face antérieure et 17 sur la face postérieure, mais seules 16 pointes sont encore discernables, une cassure ayant provoqué un enlèvement important dans l'angle distal droit.

La partie proximale a été aménagée par des ailettes latérales dont il ne subsiste que celle de gauche. L'ailette droite a été cassée à sa base ce qui a sans doute provoqué l'abandon de la pièce, soit au moment de sa fabrication, soit en cours d'utilisation (ce qui impliquerait que la fracture distale soit postérieure). Les ailettes sont marquées par une gorge transversale externe et une protubérance interne. Cette dernière est dessinée par l'encoche de fixation principale réalisée par au moins trois perforations consécutives dont les traces sont bien visibles et donnent cet aspect " trilobé ». 


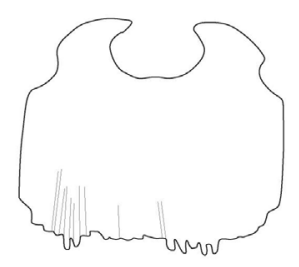

G19-6

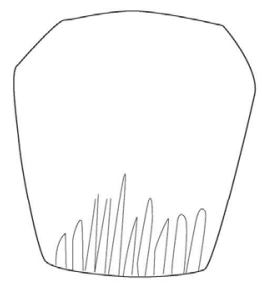

F17-5

Figure 9. - Peignes à tatouer découverts sur le site de Vaito’otia, Huahine (Société)
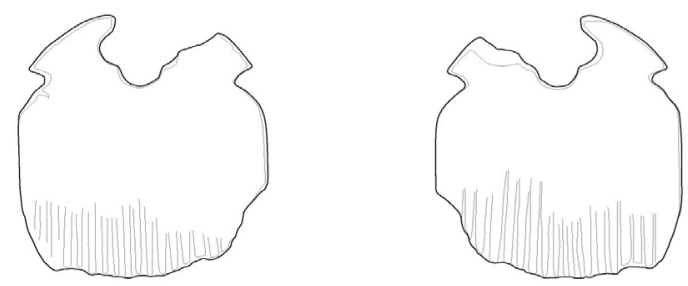

$1 \mathrm{~cm}$

FIGURE 10. - Peigne à tatouer découvert sur le site de Rivnac, Tahiti (Société)

\section{Atiahara (Tubuai, Australes), $n^{\circ} 1407$ (fig. 11)}

Ce peigne mesure $20 \mathrm{~mm}$ de long pour $9 \mathrm{~mm}$ de large, dimensions qui en font une pièce assez étroite. Elle est complète à l'exception de l'extrémité des pointes dont une seule, longue de $2 \mathrm{~mm}$, est encore visible. Les deux bords latéraux sont parallèles sauf dans le quart proximal où ils convergent vers le sommet au profil légèrement courbe. Les deux faces sont occupées par les stries de rainurage au nombre de huit qui marquent neuf pointes au total. Si les stries les plus à gauche de la face antérieure sont courtes et débutent au milieu de la pièce, celles rainurées sur la moitié droite sont beaucoup plus longues et débutent dans la partie proximale. Certaines stries sont même interrompues par le bord supérieur du peigne ce qui implique que leur réalisation a précédé l'aménagement définitif du bord proximal.

Le système de fixation est plus simple que celui décrit pour les autres objets. En effet, il s'agit d'une unique perforation centrale de $2,5 \mathrm{~mm}$ de diamètre, apparemment biconique. Elle correspond probablement aux systèmes d'encochement ou de fixation sommitale décrits dans le texte.

\section{Parcelle proche d'Atiahara (Tubuai, Australes), $n^{\circ}$ 452-318-TNP-1f (fig. 11)}

Nous n'avons eu accès qu'à un dessin de cette pièce, aussi les observations qui sont proposées dépendent-elles grandement de la qualité relative de sa représentation. La pièce mesure $19 \mathrm{~mm}$ de long et $15 \mathrm{~mm}$ de large. Seule une partie du bord latéral gauche est conservée, les autres bords étant cassés. Les deux faces présentent des stries de rainurage réservées au tiers distal. On en compte neuf sur la face antérieure et 12 sur la face opposée (le délitage de la nacre dans l'angle distal droit a fait disparaitre trois stries). La superposition des deux faces montre que l'objet en comportait au moins 13 avant sa cassure. Leur extrémité a désormais disparu. La partie proximale, elle aussi brisée, ne présente aucune trace d'aménagement et interdit, de fait, de comprendre le mode de fixation prévu.

\section{Parcelle proche d'Atiahara (Tubuai, Australes), pas de numéro d'inventaire (fig. 11)}

Ce fragment de peigne à tatouer de $17 \mathrm{~mm}$ de long et $14 \mathrm{~mm}$ de large est très incomplet. Il ne subsiste que la partie distale suite à une cassure franche transversale. Cette fracture, qui a rendu l'objet inutilisable, est survenue après la mise en forme des pointes (seules deux extrémités sont bien conservées). Le bord gauche est conservé et semble droit par rapport à l'axe des stries. Une rupture apparaît peut-être en partie proximale où il s'oriente vers le sommet. On compte 12 stries sur une face et 13 sur l'autre, soit au moins 14 pointes. Une différence est notable entre les deux faces : sur l'une, les stries sont courtes et régulières tandis que sur l'autre, elles sont plus longues et même interrompues par la ligne de fracture. La strie située la plus à gauche de la face interne n'a pas atteint le bord distal. Pourtant la 


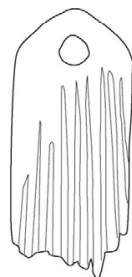

\#1407
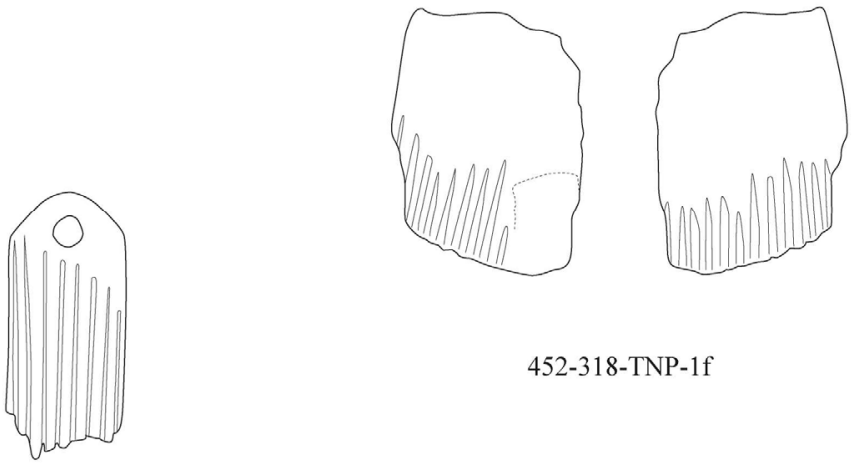

452-318-TNP-1f
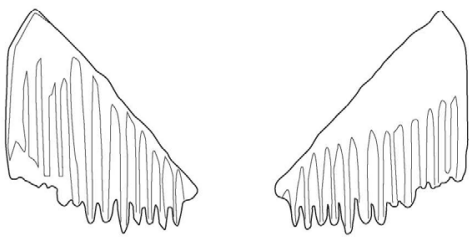

non identifié

FIG. I I. - Peignes à tatouer découverts à Tubuai (Australes)

pointe qu'elle devait dessiner est presque formée par la strie réalisée sur la face opposée. Ainsi, on pourrait supposer que la fracture mésiale est survenue au cours de cette étape, entraînant l'abandon de la pièce. Notons que les stries de la face postérieure sont particulièrement larges par rapport aux autres pièces du corpus et ce, dès le point de départ du rainurage, ce qui signale un geste très appuyé.

\section{Remerciements}

Nous tenons à remercier tout particulièrement le Dr. Y. Sinoto qui nous a transmis les photographies des peignes trouvés par lui sur le site de Hane, ainsi que L. Miller pour les informations partagées à propos des exemplaires de Tubuai. Nous souhaitons également remercier T. Hiquily, conservateur adjoint du musée de Tahiti et des îles ainsi que T. Maric du Service de la Culture et du Patrimoine qui nous ont ouvert les portes des réserves afin de photographier les objets qui y sont conservés. Nous remercions enfin A. Hermann pour ses avis et $\mathrm{S}$. Galliot pour les compléments d'information qu'il nous a apportés.

\section{BIBLIOGRAPHIE}

Ambrose William, 2012. Oceanic Tattooing and the Implied Lapita Ceramic Connection, Journal of Pacific Archaeology 3 (1), pp. 1-21.
Best Eldson, I904. The Uhi-Maori, or Native Tattooing Instruments, Journal of the Polynesian Society 13, pp. 166-172.

Buck Peter H. (Te Rangi Hiroa), 1927. The Material Culture of the Cook Islands. New Plymouth, Mem. Board of Maori Ethnological Research 1.

-, 1930. Samoan Material Culture, Honolulu, B. P. Bishop Museum Bulletin 75 .

—, 1938. Ethnology of Mangareva, Honolulu, B. P. Bishop Museum Bulletin 157.

-, 1949. The coming of the Maori, Wellington, Maori Purposes Fund Board.

Carlier Christelle, 2002. Étude typologique de la collection d'hameçons du site de $\mathrm{Ma}$ nihina, Ua Huka (îles Marquises), mémoire de maîtrise, Université Paris-I Panthéon-Sorbonne, $88 \mathrm{p}$.

Carlier Christelle et Éric Conte, 2oio. Propositions de nouvelles approches dans l'étude des hameçons océaniens, Journal de la Société des Océanistes 128, pp. 133-146.

Davidson Janet M., I984. The Prehistory of New Zealand, Auckland, Longman Paul.

Eddowes Mark et John K. Dennison, 1996. Sauvetage archéologique du site de TaitapuRivnac, Bulletin de la Société des études océaniennes 272, pp. 3-23. 
Emory Kenneth P., 1975. Material Culture of Tuamotu Archipelago, Honolulu, Pacific Anthropological Records 22.

Galliot Sébastien, 2011. Pe’a et Malu : Le Tatouage à Samoa (1722-2010). Technique et culture dans une société de Polynésie Occidentale en mutation, Paris, thèse de doctorat de l'ehess, 3 vols (418 p., 43 p., 142 p.)

Handy Edward S. C., 1923. The Native Culture in the Marquesas, Honolulu, Bernice P. Bishop Museum Bulletin 9.

KIrch Patrick V., David W. Steadman, Virginia L. Butler, Jon Hather and Marshall Weisler, 1995. Prehistory and human Ecology in Eastern Polynesia: Excavations at Tangatatau Rockshelter, Mangaia, Cook Islands, Archaeology in Oceania 30, pp. 47-65.

Mead Sidney M., 1968. Polynesian Hafted Adzes: A comparative study of Form and Decoration, Carbondale, thèse de doctorat, Southern Illinois University.

—, I971. An Analysis of Form and Decoration in Polynesian Adze Hafts, Journal of the Polynesian Society 80 (4), pp. 485-496.

Molle Guillaume, 2011. Ua Huka, une île dans l'Histoire. Histoire pré- et post-européenne d'une société marquisienne, Tahiti, thèse de doctorat de l'Université de la Polynésie française, 2 vols., 432 p.

Otтino Marie-Noëlle et Pierre Otтino, 1999. Te Patu Tiki, l'art du tatonage aux îles Marquises, Tahiti, Éditions Ch. Gleizal, 303 p.

Pendergrast Michael, 200o. Tikopian Tattoo, Auckland, Bulletin of the Auckland Museum 18.

Robitaille Benoît, 2007. A preliminary typology of perpendiculary hafted bone tipped tattooing instruments: Toward a technological history of Oceanic Tattooing, in C. Gates St-Pierre and R.B. Walker (eds), Bones as tools: Current methods and interpretations in worked bone studies, Oxford Archaeopress, BAR International Series 1622, pp. 159-174.

Rolett Barry V. and Eric Conte, 1995. Renewed Investigations of the Ha'atuatua dune (Nuku Hiva Marquesas Islands): a key site in Polynesian Prehistory, Journal of the Polynesian Society 104 (2), pp. 195-228.

Roтн H. Ling, I905. Tatu in the Society Islands, The Journal of Anthropological Institute of Great Britain and Ireland 35, pp. 283-294.

Sinoto Yosihiko, 1966. A Tentative Prehistoric Cultural Sequence in the Northern Marquesas Islands, French Polynesia, Journal of the Polynesian Society 75 (3), pp. 286-303.

—, 1979. Excavations on Huahine, French Polynesia, Pacific Studies 3 (1), pp. 1-40.

-, 1983. Archaeological Excavations on the Vaito'otia and Fa'ahia sites on Huahine Island, French Polynesia, National Geographic Society Research Reports 15, pp. 583-599.

Sinoto Yosihiko et Marimari Kellum, 1965. Preliminary Report on Excavations in the Marquesas Islands, French Polynesia, B. P. Bishop Museum Polynesian Archaeological Program, $55 \mathrm{p}$.

vON DEN SteInen Karl, 2005 [1925-1928]. Les Marquisiens et leur art: l'ornementation primitive des mers du Sud, vol.2: Plastique, Papeete, Musée de Tahiti et des Îles, Éd. Le Motu, 292 p.

Worthy Trevor H. and Robert Bollt, 2011. Prehistoric Birds and Bats from the Atiahara Site, Tubuai, Austral Islands, East Polynesia, Pacific Science 65 (1), pp. 69-85. 